PROC. OF JSCE

No. 332, April 1983

\title{
AUTOMATED STRUCTURAL TESTING USING MICROCOMPUTER SYSTEM
}

\author{
By Yoshiji NIWA*, Eiichi WATANABE** \\ and Hidenori ISAMI***
}

\section{INTRODUCTION}

The current engineering advancements may be well characterized by the unprecedented developments of LSI in the field of electronics industry. Today, a great number of microcomputer models are being produced with excellent LSI chips and are available in the market to answer rapidly growing needs. For example, the more and more testing facilities are being accommodated with microcomputers.

On the other hand, structural engineers have been facing many intricate problems including nonlinear structural behaviors. Thus, the subjects of nonlinear structural behaviors have been increasingly attracting researchers' attention, and struggles for the better assessment of the safety of structures appear to have become even more significant. An example of such problems can be found in thin-walled steel members which are frequently being used for civil engineering structures, especially for bridges. The loadcarrying capacity of such thin-walled members, nevertheless, are supposedly liable to the initial deformations and eccentricities ${ }^{1}$.

For such a reason, the Society of Steel Construction of Japan, JSSC, has conducted an extensive survey in the recent years involving the measurement of the initial imperfections of actual steel bridges and their statistical analyses ${ }^{2), 3)}$. These initial imperfections, however, have been measured during the temporary assembly of the bridge superstructures at workshops. Thus, they may progressively grow with time as a result of elasto-plastic large deformation process and gradual adverse deterioration may result in under the stochastic loading process

* Member of JSCE, Dr. Eng., Professor.

** Member of JSCE, Ph. D., Associate Professor, Kyoto University.

*** Member of JSCE, M. S., Research Associate, Kohchi Technical College. in the lifetime of structures). The nature of such a problem is necessarily nonlinear in both senses of geometry and material. Thus, the experimental verification may be found not easy either, since the control of a nonlinear system is in general quite difficult and laborious.

This report thus presents a testing system using a microcomputer as its core, and a technique is reported on the precise detection of the static load-carrying capacity and the teteriorating properties of thin-walled structural components subjected to repetitive loading cycles involving elasto-plastic large deformations. A series of buckling tests have been conducted so far on steel stub columns with cross sections of cruciform, cylindrical shell, and box with or without the longitudinal ribs under repetitive static loading.

The testing system reported herein is shown in Fig. 1 to illustrate the schematic construction, and is so designed that the following tasks are accomplished:

(a) easy and efficient measurement of initial and residual deflections on the surfaces of the test specimen,

(b) quick real-time acquisition and processing of large quantities of the data for the swift decision making on the continuously varying state of the test specimen,

(c) digital feedback control of the servo testing machine,

(d) real-time display of the performance of the specimen,

(e) efficient storage of the acquired data set, and

(f) transmission of the data to a large computer center for further large-scale computations via intelligent TSS terminal.

This testing procedure may be in good contrast to the so-called on-line "hybrid test" procedure. The former aims at the evaluation of the more fundamental load-carrying characteristics of the specimen under the loading in ramp wave forms controllable with digital feedback of the servo testing machine, and further- 


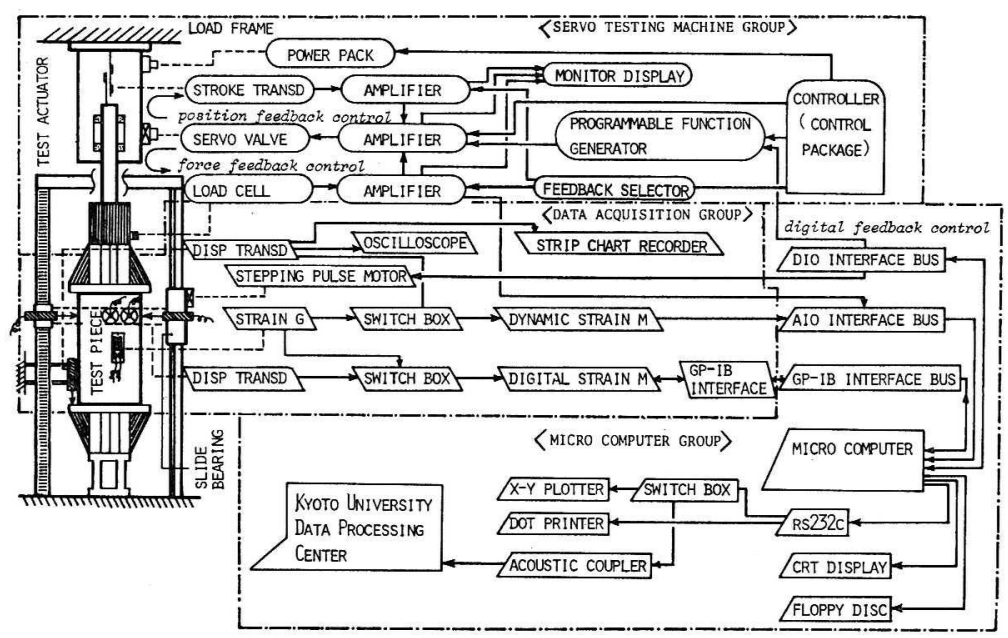

Fig. 1 Automated Testing System.

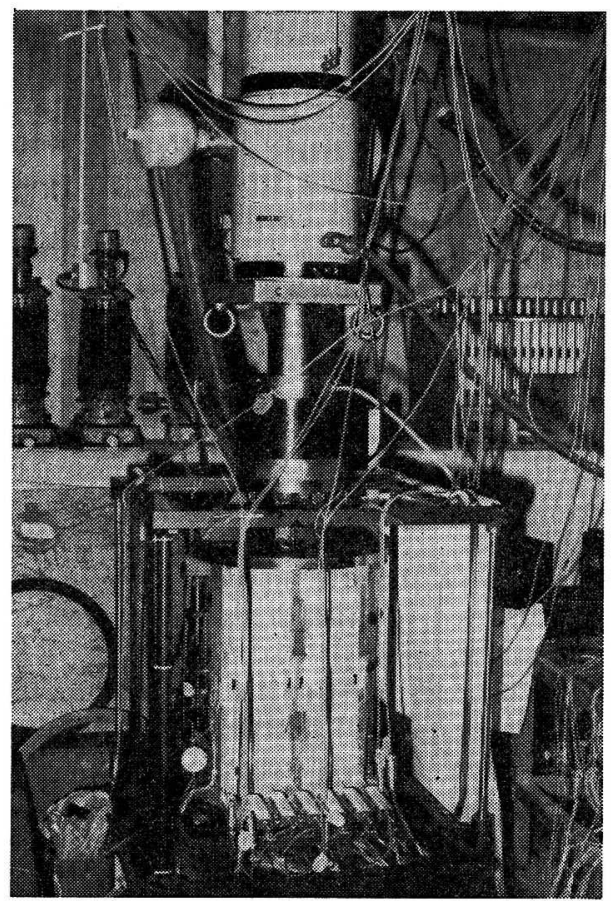

Photo 1 Servo Testing Machine and a Stab Column.

more, the aquisition and processing of large quantities of data. The latter, on the other hand, employs the input acceleration wave records of ground motions either actual or simulated, to prescribe the loading, and moreover, involves the integration of the equations of motion of the simplified model of the specimen with respect to the time to solve for the displacements so as to be assigned as the input to a servo-controlled testing machine ${ }^{5)}$.

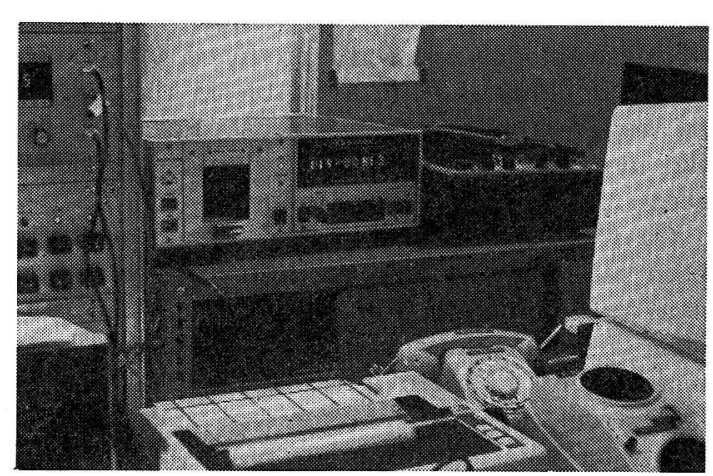

Photo 2 Data Acquisition Devices at Work.

From Fig. 1, it will be seen that the system consists of the following three groups: SERVO TESTING MACHINE GROUP, DATA ACQUISITION GROUP, and MICROCOMPUTER GROUP. Photo 1 shows a snapshot showing the actuator, servovalve, accumulater, load cell, and a rig for measuring deflections on the surfaces of the test specimen during a box stub column test. Also shown in Photos 2 and 3 are the data acquisition devices, and another snapshot of the microcomputer during such a test, respectively.

These three groups will be described in the subsequent chapter in detail as the basic hardware of the testing system. Since as far as microcomputers are concerned, the I/O (Input/Output) control through the interfaces can be most efficiently carried out by use of BASIC (Beginner's All-Purpose Symbolic Instruction Code) language, it is used throughout the software developed herein. Thus, some application programs for the use of several interfaces will be 


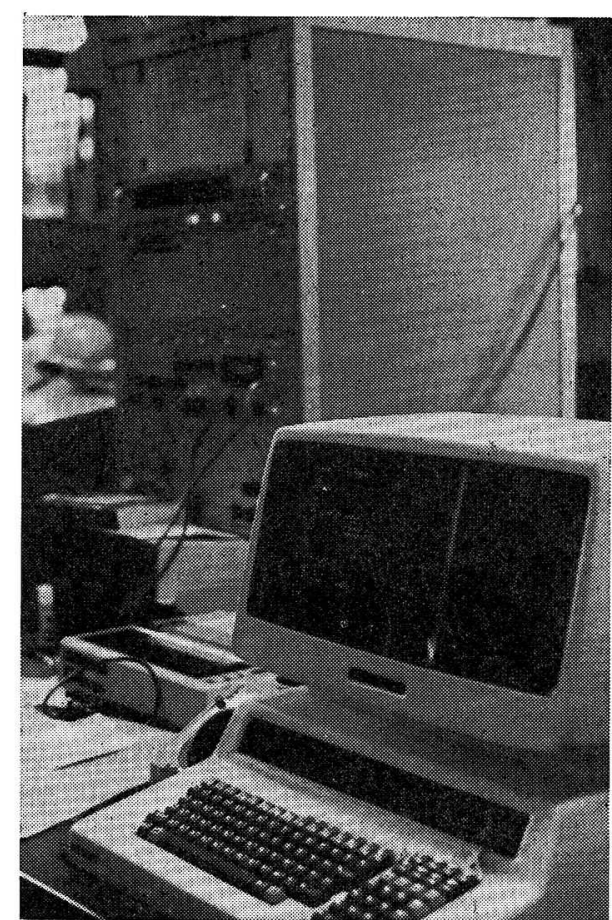

Photo 3 Microcomputer System at Work.

provided also for illustrative purposes.

Thenceforth, a number of key points will be reported on the software developed herein including the schematic flow chart of the basic programs. The software includes the method for detecting the local load-carrying capacity of the destructive portion of the specimen, generation of pulse signals to the function generator of the testing machine for its digital feedback control, and the data acquisition. At the end of this report, some results of data processing will be provided for demonstrative purposes.

As mentioned earlier, the three groups of the hardware will be described in what follows.

\section{HARDWARE OF TESTING SYSTEM}

\section{(1) Servo Testing Machine Group}

This group refers to the servo-controlled electrohydraulic closed-loop system, and is shown in Fig. 2. It mainly consists of the power pack, hydraulic actuator, and several controllers. The actuator is operated through the servo controller according to the prescribed voltage from the function generator: It accepts two inputs, one being the command signal from the function generator, and the other being a system analog feedback signal of either load, or stroke; while it generates one principal output, the control signal to the servovalve.

The function generator used herein is an 8-bit microprocessor programmable upto 16 steps. In each step, either of the sine, triangle, ramp, or hold wave form can be selected. Fig. 3 shows an example of combination wave forms through the function generator.

Furthermore, in the proposed testing system, a digital feedback is accomplished as follows: The function generator is pre-programmed to achieve either the constant stroke velocity or constant load rate, and is externally controlled by negative pulse signals of TTL level* through the digital Input-Output interface of the microcomputer.

* The TTL (Transistor-Transistor Logic) level is so selected that when the power supply is 5 Volts, the signal level greater than or equal to 2.4 Volts is regarded as "High", and the one less than or equal to 0.4 Volt as "Low".

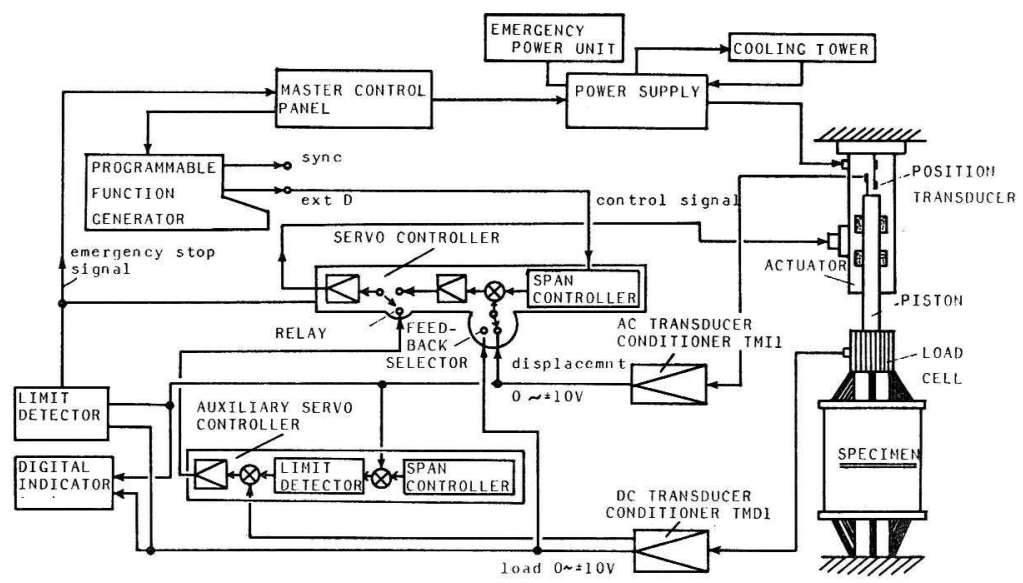

Fig. 2 Servo-controlled Electrohydraulic Testing Machine. 


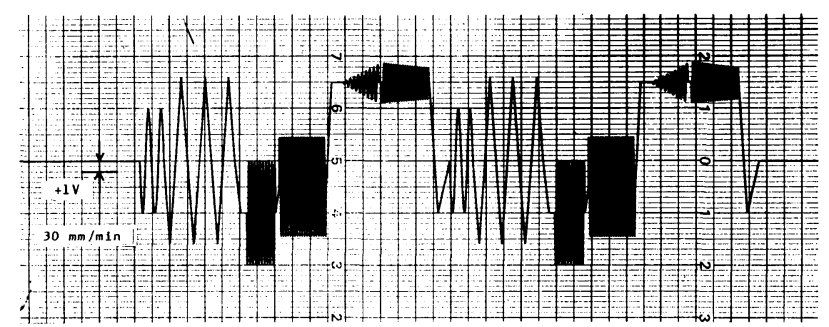

Fig. 3 An Example of Combination Wave Forms by Function Generator.

\section{(2) Data Acquisition Group}

The response of the structures subjected to the servo-controlled load or the stroke displacement is detected by the devices of this group. These data are processed in real time so that they can be used as the digital feedback data, recorded in floppy disks, and displayed by the microcomputer. The group consists of the following devices: displacement transducers, digital strain amplifiers, dynamic strain amplifiers, hold-type switch box, GP-IB, pen strip chart recorder, oscilloscope, and a motor-driven rig for displacement transducers.

In the proposed testing system, strains and displacements are subjected to the data acquisition. These readings are sensored and amplified using dynamic strain amplifiers and a digital strain amplifier. For a successful digital feedback control, the real time and continuous data acquisition is mandatory. Thus, in the system proposed herein, many dynamic strain amplifiers are used to make the real time feedback possible, since the data

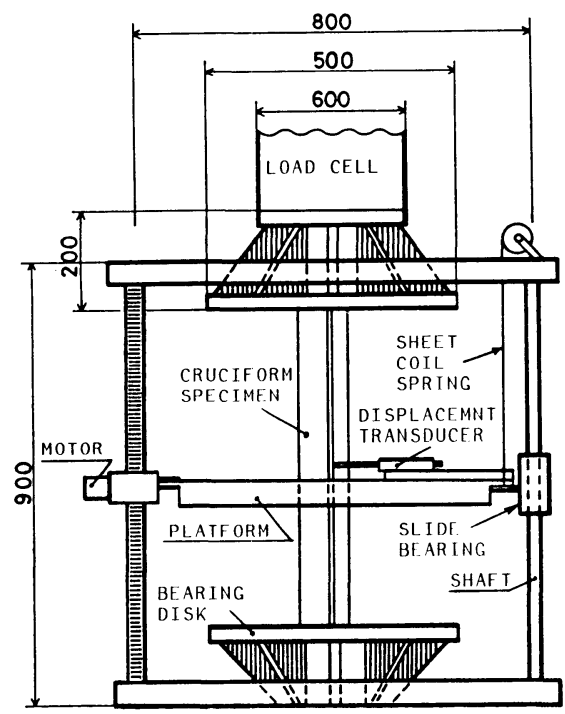

Fig. 4 Rig for Measuring Displacements. acquisition by means of the digital strain amplifier would have taken more seconds. The digital strain amplifier, however, is used to obtain the spatial distribution of the displacements of the test specimen.

Moreover, Fig. 4 shows the rig especially manufactured for displacement transducers. This rig consists mainly of four columns, of which one pair of the diagonals have slide bearings; while another has parallel grooves for the operation of motor-driven platform equipped with the displacement transducers.

\section{(3) Microcomputer Group}

This group has a microcomputer as its core, and plays the key role as the system controller of the testing system. The microcomputer system used is illustrated in Fig. 5, and is seen to consist of CPU (Central Processing Unit), main memory,

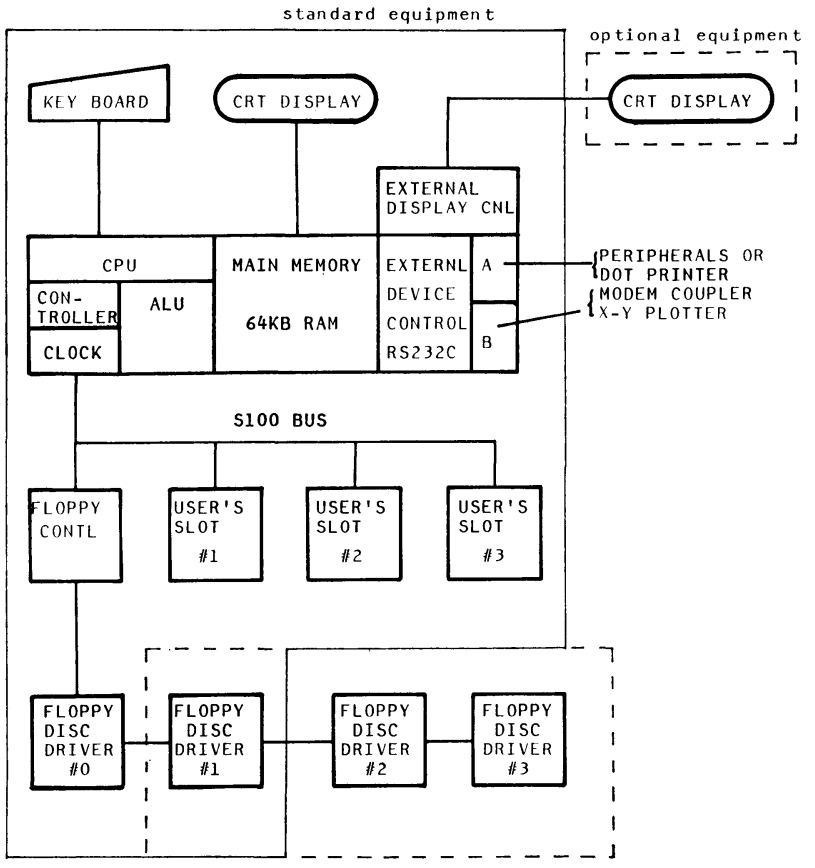

Fig. 5 Brief Description of Microcomputer System Being Used. 
and peripherals such as dot printer, CRT display, keyboard, disk driver, and a MODEM coupler for the data transmission to a larger computer center $\left.\left.^{6}\right), 7\right)$.

For efficient data transmission of data among these devices, several interfaces are required ${ }^{6), 8)}$. These include the Analog Input/Output (AIO) interface for the analog to digital transform, or vice versa; Digital Input/Output (DIO) interface for the real-time control of the programmable function generator of the servo-controlled testing machine; and finally, General Purpose Interface Bus (GP-IB), for the digital data.

\section{DESCRIPTIONS ON INTERFACES USED}

\section{(1) Analog Input/Output (AIO) Interface}

In the testing system presented herein, the functions of the analog $\mathrm{I} / \mathrm{O}$ interface are to convert the input analog data of maximum 16 channels to the corresponding 12-bit digital data, as shown in Fig. 6, and to convert the input 8bit digital data of maximum 2 channels to the corresponding analog data ${ }^{9}$.

The analog data plays such an important role of the real-time control of the testing machine, that the calibration of $\mathrm{A} / \mathrm{D}$ converter must be performed precisely. Thus, a method of the calibration is briefly given in the following: The analog input data, ranging from -10 Volt to +10 Volt, may be conveniently converted to decimal numbers from 0 to $4095\left(=2^{12}-1\right)$. Now, when -10 Volt is given as the input to channel

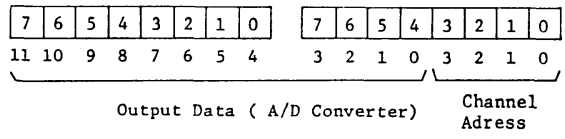

Fig. 6 12-Bit Digital Data Corresponding to Analog Input.

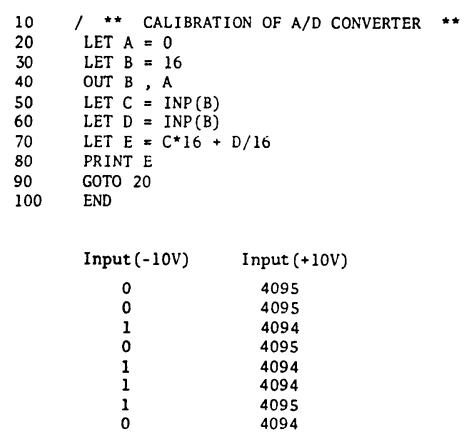

Fig. 7 Program for Calibration of A/D Converter and Its Result on CRT Display.
0 , the $A / D$ converter can be adjusted so that its output would become 0 or 1 with equal probability of $50 \%$ in order to keep the error within 1 bit. Next, when +10 Volt is given as the input to channel 0 again, it can be adjusted so that its output becomes either 4094 or 4095 with equal probability of $50 \%$.

Fig. 7 shows the calibration program written in a simple Extended BASIC language, together with the result on CRT display. The processing time for analog input is about 110 microseconds a channel.

\section{(2) Digital Input/Output (DIO) Interface}

In the proposed testing system, the function of the digital I/O interface is to handle input and output 32-bit data parallel, respectively using TTL level and the negative logic. The interface possesses both 4 input and 4 output ports, where each port can take care of 8-bit data parallel and two control lines, simultaneously ${ }^{9}$.

This interface is used in the system in order to control a programmable function generator: A negative pulse signal with the duration of 10 msec or more is transmitted as the input to either of the four external control terminal pins of FORWARD, RESET, HOLD, and RUN. Fig. 8 and Table 3 illustrate the generation of the negative pulse signal $\bar{c}_{\boldsymbol{b}}(1)$ to be given as input to either of the lower 4 bits of DIO output pins for the assigned decimal number $c(1)$.

An example of a simple Extended BASIC program is illustrated in Fig. 9 to trigger the function generator pre-programmed according to Table 2. The generated wave form is latched by the AIO interface, and the result is provided also herein. It will be seen to consist of a ramp wave for loading and another ramp wave for unloading.

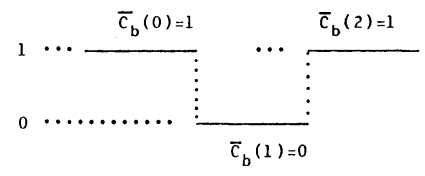

Fig. 8 Digital Input Pulse to the Selected Bit.

Table 1 Array of the Signal $\bar{c}_{b}(1)$ for Input Decimal Number $c(1)$.

\begin{tabular}{l|c|c|c|c|c}
\hline \multirow{2}{*}{$\begin{array}{c}\text { Ext. } \\
\text { Control }\end{array}$} & \multirow{2}{*}{$\begin{array}{c}\text { Decimal } \\
\text { Number } \\
c(1)\end{array}$} & \multicolumn{3}{|c|}{ Signal $\bar{c}_{b}(1)$ to Binary Bits } \\
\cline { 3 - 6 } & 1 & $2^{3}$ & $2^{2}$ & $2^{1}$ & $\begin{array}{c}\text { (LSB) } \\
2^{0}\end{array}$ \\
\hline Forward & 2 & 1 & 1 & 1 & 0 \\
Reset & 4 & 1 & 0 & 1 & 1 \\
Run & 8 & 0 & 1 & 1 & 1 \\
Hold & & & & & 0 \\
\hline
\end{tabular}


I ** TEST PROGRAM OF AIO AND DIO INTERFACES * DIM $\mathrm{B}(0), \mathrm{P}(7), \mathrm{C}(2), \mathrm{A}(20), \mathrm{B} 1(100)$

/ Parameters for Analog Input

LET $A(1)=1 \quad-\quad$ Device Number

LET $A(2)=1 \quad-\quad$ No. of Data

LET A(3) $=-1 \quad-\quad$ Interval ( $1 \mathrm{sec}$ )

LET $A(4)=0 \quad-$ Auto Start

LET $A(5)=0 \quad-\quad$ Channel Number

LET A(6) $=-1 \quad--\quad$ End of Channels

/ Parameters for Digital Output

LET $P(1)=0 \quad--$ Device Number

LET $P(2)=1 \quad-\quad$ Port Number

LET $P(3)=3 \quad$-. No. of Data

LET $P(4)=1 \quad-\quad$ Negative Logic

LET $P(6)=1 \quad-$ Interval (1msec)

LET $P(7)=0 \quad--\quad$ Auto Start

/ Data of Digital Output

LET C $(0)=0$

LET $\mathrm{C}(2)=0$

/ Digital Output ( Binary)

/ ... RUN --

LAT C (1) $=4$

CALL \#2 $, \mathrm{C}, \mathrm{P}$
LET I = 0

Analog Input ( A/D Converter)

CALL \#5, B, A

LET $I=I+$

LET $B 1(I)=B(0) \star 0.005-10.24$

IF I $<50$ THEN GOTO 210

LET $N=I$

FOR $I=1$ TO N

PRINT I, B1(I)

NEXT I

END

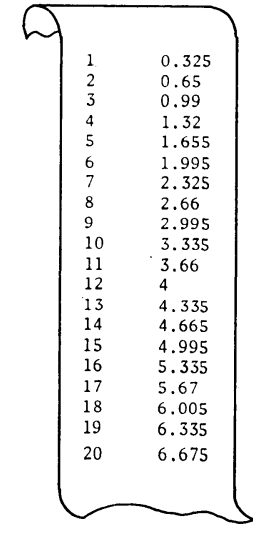

DISPLAY OF RESULTS

ON CRT THROUGH AIO.

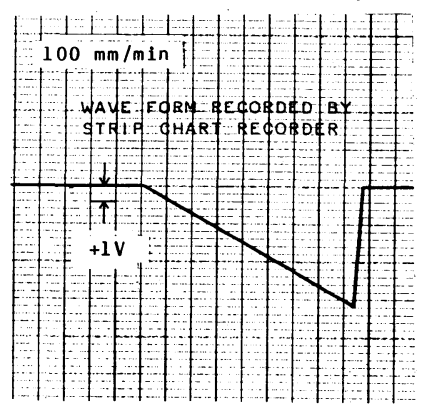

Fig. 9 An Example Program to Trigger Function Generator through DIO Interface and Acquisition through AIO Interface.
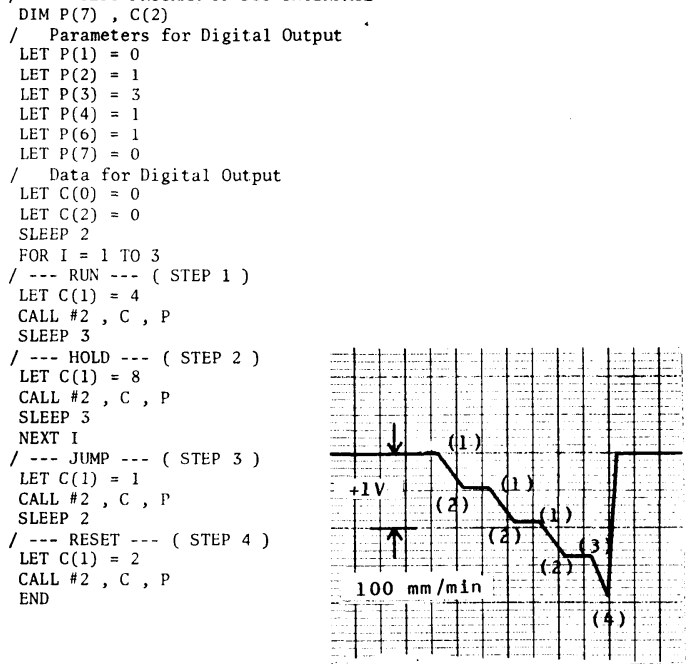

WAVE FORM BY STRIP CHART RECORDER

Fig. 10 Another Example Program to Trigger and Hold Function Generator through DIO Interface.
Table 2 An Example Program of Function Generator to Generate Ramp Wave Form.

\begin{tabular}{c|l|c|c}
\hline Step & Function & $\begin{array}{c}\text { Rate } \\
\text { (sec/FS) }\end{array}$ & $\begin{array}{c}\text { Final } \\
\text { (FS in Volt) }\end{array}$ \\
\hline 0 & Ramp & 25 & 8 \\
$1 \sim 14$ & Skip & - & - \\
15 & Ramp & 1 & 0 \\
\hline
\end{tabular}

Table 3 Another Example Program of Function Generator to Generate Ramp Wave Form.

\begin{tabular}{c|l|c|c}
\hline Step & Function & $\begin{array}{c}\text { Rate } \\
(\mathrm{sec} / \mathrm{FS})\end{array}$ & $\begin{array}{c}\text { Final } \\
\text { (FS in Volt) }\end{array}$ \\
\hline 0 & Ramp & 12 & 2 \\
1 & Ramp & 24 & 8 \\
$2 \sim 14$ & Skip & - & - \\
15 & Ramp & 1 & 0 \\
\hline
\end{tabular}

Furthermore, another example of $\mathrm{Ex}$ tended BASIC program is also illustrated in Fig. 10 both to trigger and hold the function generator pre-programmed according to Table 3. The generated wave form is recorded by the strip chart recorder and is also shown herein.

\section{(3) GP-IB (General Purpose Interface Bus) $)^{9) \sim 12)}$}

GP-IB is referred to as a standardized bus for transmission of parallel 8-bit data recognized as an IEEE-Standard in U.S.A. The standard defines the shape of connectors, pin arrangements, their functions, timing of signals, and so forth. The interface possesses the following characteristics:

(a) complete standardization,

(b) easy connections using a connector cable with other devices totalling maximum number of 14 ,

(c) handshake method of data transmission, and,

(d) communication lines consist of 5 control lines and 3 handshake lines in addition to 8 data lines.

The interface may be called either HPIB, IEEE-488 Bus, or IEC Bus (25 pins). The 8-bit data may be transmitted on the bi-directional bus in parallel. The data transfer contains three types: firstly, from a certain device to a microcomputer, secondly, from a microcomputer to several 
devices, and thirdly, among devices. In the proposed testing system, a GP-IB was used merely for the data acquisition through the digital strain amplifier.

Devices on GP-IB System

The devices forming GP-IB system may be operated with the following functions.

(a) TALKER: to transfer data to other devices. There may not be plural talkers simultaneously on a GP-IB line.

(b) LISTENER: to accept data from other devices. There may be many listeners at a time.

(c) CONTROLLER: to control the data transfer among devices connected with GP-IB line. The CONTROLLER may address a device as a TALKER and some devices as LISTENERS. There may be plural controllers: however, only a single controller can be active at a time.

GP-IB Bus Lines

The GP-IB consists of 24 bus lines: 16 signal lines and 8 ground lines. Eight of 16 signal lines are data lines, which can transfer the input output data, address, control, and status among devices connected. The data transfer is bit parallel, byte serial. 'L"' level of ATN on Control Lines is called Command Mode, then, the internal control signal is transmitted among devices from a controller. If ATN is on " $\mathrm{H}$ " level, then byte data may be transferred from a talker to listeners, and this case is called Data Mode.

Three Transfer Lines are for controlling the data transfer on Data Lines. The way of the data transfer is hand-shaking. This is, the data can be transferred at the lowest rate of devices. Handshaking

Fig. 11 illustrates the timing chart of threeline handshake. Here, several listeners are assumed to accept data from a talker. Detailed descriptions are provided subsequently according to the encircled number in the figure:

(1), (2): Initial state of the data transfer is $\mathrm{DAV}=$ "H", NRFD="L", and NDAC="L".

(3): Confirming the initial state, a talker transfers one byte data on Data Lines.

(5): NRFD remains " $L$ " level until all listenners get ready for accepting data; then, NRFD becomes "H" level.

(3) (6): Talker, confirming $\mathrm{NRFD}=$ " $\mathrm{H}$ ", makes DAV= "L" after certain time period (4).

(6) (8): Listeners can accept data on Data Lines while NRFD = "L". NDAC will not become " $H$ " level unless all listeners finish accepting data.

(8) (10): Talker, confirming $\mathrm{NDAC}=$ " $\mathrm{H}$ ",

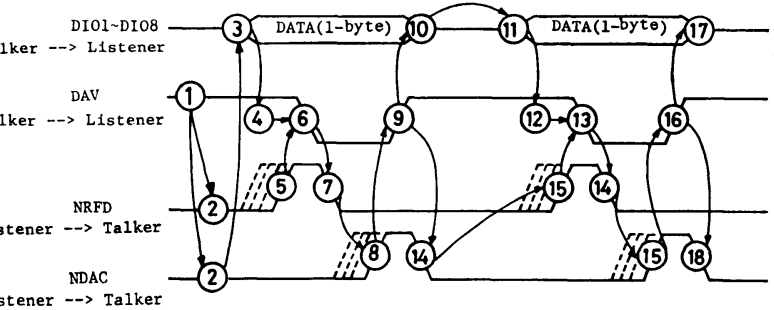

Fig. 11 Timing Chart of Three-line Handshake.

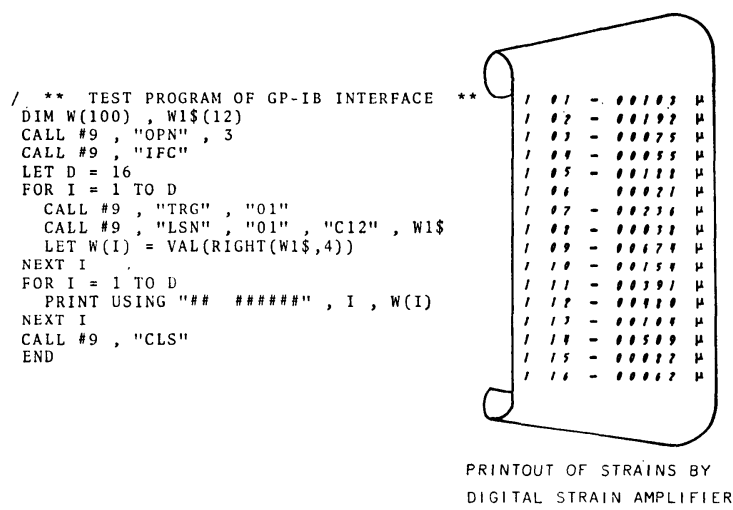

Fig. 12 Test Program of GP-IB and Its Output.

i.e., that the data transmission of one byte length has terminated, makes DAV $=$ " $\mathrm{H}$ ".

(II): Talker transfers the next byte data on Data Lines in the similar manner, and so on.

A simple example to use a GP-IB for the digital strain amplifier will be given here through the use of Extended BASIC program as is shown in Fig. 12. Here, the talker is the digital strain amplifier and the listener is the microcomputer. This example shows how to acquire 16 digital strains, and several definitions of the terms in the program will be explained in the following:

OPN: open the function of GP-IB,

CLS: close the function of GP-IB,

LSN: accept data from some device(s) adressed,

TRG: trigger devices,

VAL (RIGHT(W1\$, 4)): change character variable $\mathrm{W} 1 \$$, from the 4 th column to the 12 th, to the corresponding numerical variable.

\section{PROGRAMMING IN BASIC LANGUAGE}

\section{(1) Flow Chart}

One of the objectives of the proposed testing system is to facilitate finding the correlation of of the load-carrying capacity of structures with their initial imperfections. So far, most of the 
researchers have conducted such ordinary tests, as to obtain only a single load-carrying capacity for a specimen. In the present testing system, on the other hand, the more data are obtained in the following process: for a single specimen, the initial deformations were firstly measured, and then the pecimen was subjected to the longitudinal compressive loading, using ramp wave forms through the function generator and unloading using another ramp wave again, upon the detection of the local maximum load, i.e., the load-carrying capacity. Finally, the residual deformations are measured in the same way as for the initial deformations. These residual deformations are regarded as a new set of initial deformations for the succeeding loading cycle. Then, a great number of data may be acquired in such a repetitive process for a single specimen.

Test specimens are stub columns including the cross sections of cruciform, cylindrical shell, and box with or without a longitudinal stiffener. Box and cruciform columns are designed so that the failure would occur in the form of local buckling of a pair of wider plates before a global buckling as a column. All of the specimens are shown in Fig. 13. Here, the pair of plates vulnerable to local buckling are referred to as the destructive portion and the remaining pair of plates as the non-destructive portion.

Now, let $P_{t}, P_{n d}$, and $P_{d}$ be defined as the total load, the load carried by the non-destructive portion, and the load carried by the destructive portion, respectively. And let $S$ represent the longitudinal displacement (stroke).

Using the strain readings measured on the nondestructive portion, the distributions of stresses in its cross section may be obtained assuming appropriate residual stress field. Herein, the material of the specimen is assumed to be perfectly elasto-plastic, which has been confirmed already by the material tests. Thus, $P_{n d}$ can be obtained from such stress distributions. Finally, $P_{\boldsymbol{d}}$ is determined by $P_{t}$ subtracted by $P_{n a}$. At the same time, X-Y plotter will draw the curve of $P_{\boldsymbol{d}}$ versus $S$.

In the proposed testing system, AIO and GPIB interface is adopted to obtain analog and digital data, respectively; while, DIO interface is to control the function generator operating the servo testing system.

Fig. 14 indicates the setup of the software of the present testing system.

\section{(2) Details of Software}

\section{BASIC Program}

Four BASIC programs: MAIN, START, DISP, and KLOAD, are illustrated in Fig. 15 (1) (e) (PLOT is omitted.), which form the program of automated structural testing system presented. Such five BASIC programs can be run by "CHAIN" in EBASIC. CHAIN function is to interrupt the program running and to run the other program in stead.

\section{MAIN}

This program is to be jointed with the others: START, DISP, PLOT, and KLOAD. Fig. 15 (a) shows the flow chart of the program.

Here, "loading step" and "loading substep" are defined in the following: The loading step refers to the step during which the specimen is subjected to the loading and holding if the prescribed load is attained, and the measurements of displacements are performed and $\mathrm{X}-\mathrm{Y}$ plotter is operated if necessary. Whereas, the loading sub-

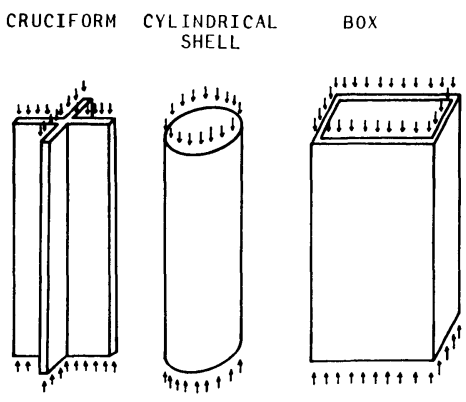

Fig. 13 Test Specimens.

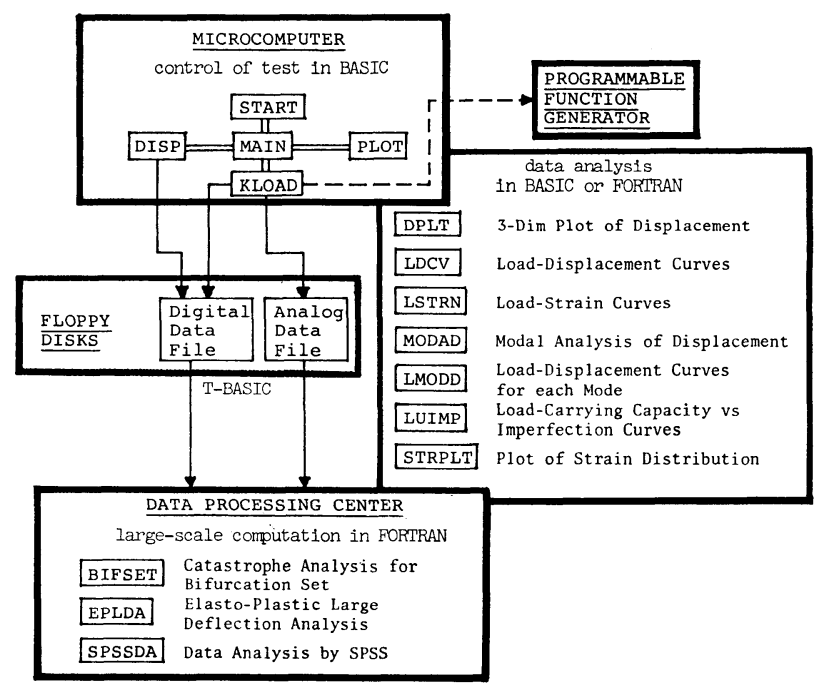

Fig. 14 Setup of Software. 


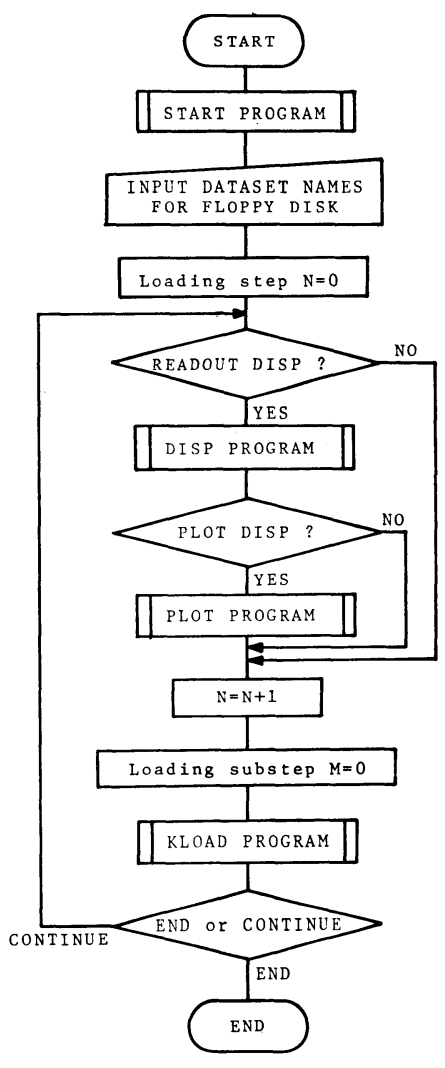

(a) Flow Chart of Main.

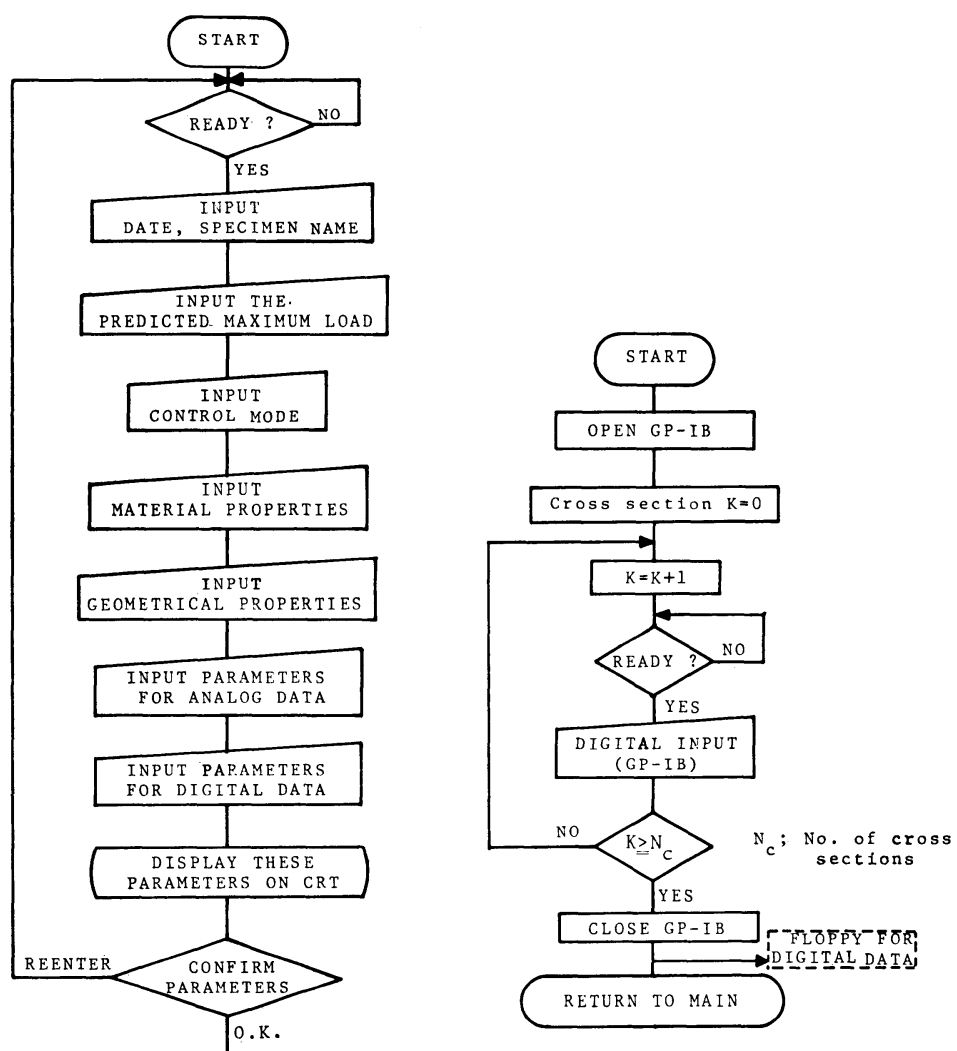

(c) Flow Chart of DISP.

RETURN TO MAIN

(b) Flow Chart of Start.

Fig. 15 (1)

step refers to the step during which the analog data are transmitted to CPU through AIO interface either in loading or in unloading.

\section{START}

This program is to input or initialize parameters after some confirmation to be ready for the test. The flow chart of the program is shown in Fig. 15 (b).

In this figure, the predicted maximum load refers to the ideal load-carrying capacity of the destructive portion, which provides a basic quantity for the decision of unloading. The control mode refers to the control either by the load or the stroke displacement of the servo testing system. Furthermore, parameters for analog or digital data refer to those of AIO, or DIO, GPIB interfaces, respectively.

DISP

This program is to measure displacements, and the flow chart is illustrated in Fig. 15 (c).

Several displacement transducers on the hori- zontal platform of the rig especially manufactured are connected with GP-IB interface in microcomputer via another GP-IB interface of the digital strain amplifier and the hold-type switch box. Digital input data can be transmitted into the floppy disk immediately.

PLOT

This program is to draw the distributions of the normalized displacements spatially on the $\mathrm{X}-\mathrm{Y}$ plotter. The flow chart is omitted.

\section{KLOAD}

This program is to select either loading or unloading in the next loading substep. The flow chart is illustrated in Figs. 15 (d) and (e).

In order to discriminate between loading and unloading in the next loading step, analog data such as the total load, $P_{t}$, the longitudinal displacements, and the strains on the destructive portion are acquired continuously and transmitted into the floppy disk.

The material of the specimen is assumed to be 


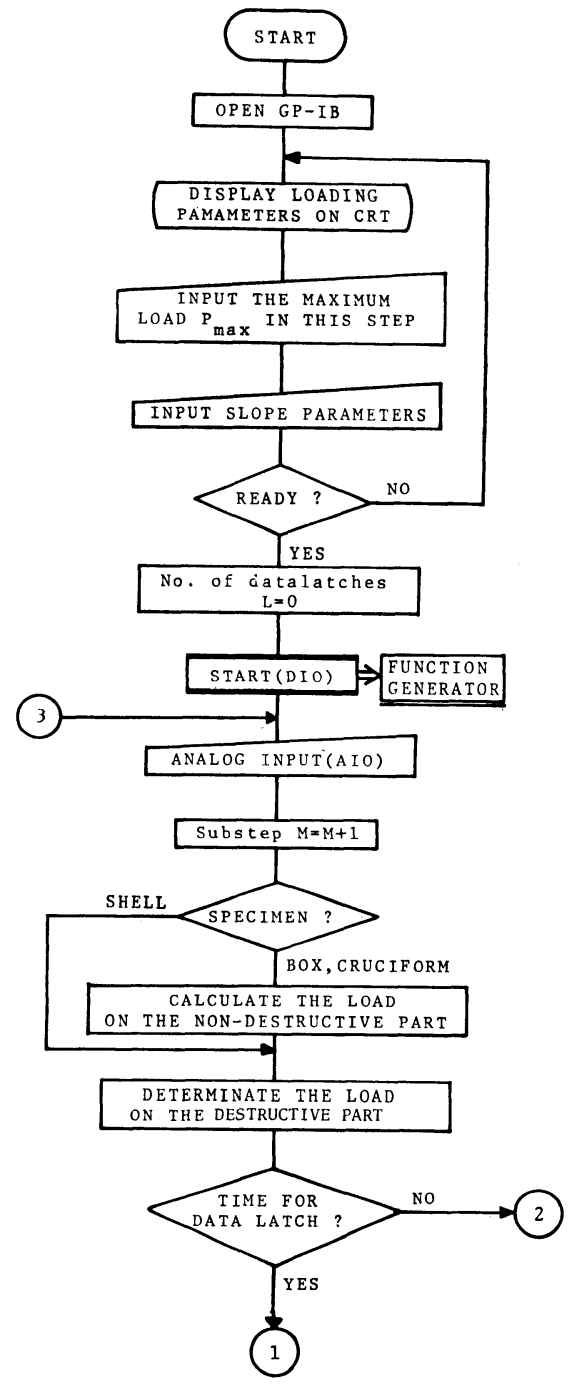

(d) Flow Chart of KLOAD.

Fig. 15 (2)

perfectly elasto-plastic. Then, the load carried by the non-destructive portion, $P_{n d}$, may be obtained from the stress distributions using the least square method. Finally, the load carried by the destructive portion, $P_{d}$, is given by,

$$
P_{d}=P_{t}-P_{n d} .
$$

In the case of cylindrical shell, $P_{d}=P_{t}, P_{n d}=0$.

To detect the local maximum value of $P_{d}$, either of the following two criteria is to be satisfied:

(a) 1st Criterion

Let $P_{M}$ and $P_{M-1}$ be the load, $P_{d}$, in the current and in the previous loading substep, respectively. Then, the variation of $P_{d}$ in each loading substep, $\Delta P$, can be defined by:

$$
\triangle P \equiv P_{M}-P_{M-1} \text {. }
$$

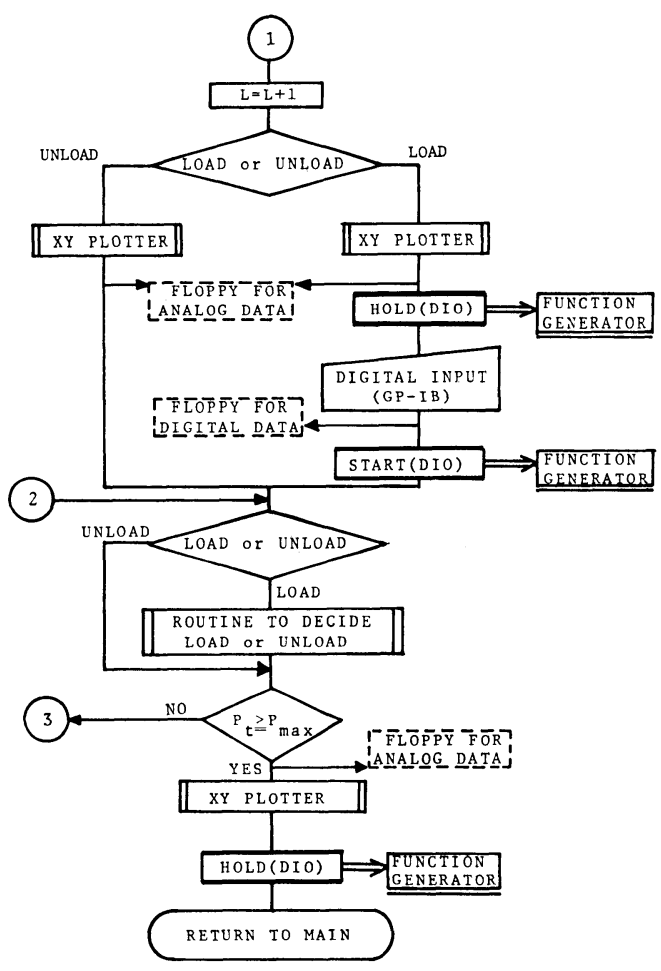

(e) Flow chart of KLOAD (continued).

Fig. 15 (3)

Then, the first criterion for the unloading may be given in a non-dimensional form for a positive small number $\epsilon$ by:

$$
\frac{\Delta P}{P_{c a}}<0 \text { and } \frac{|\Delta P|}{P_{c a}}>\epsilon .
$$

where, $P_{c a}$ refers to the static capacity of the actuator of the servo-controlled testing machine.

It is assumed that $P_{M}$ only involves errors of the servo testing machine and $\mathrm{A} / \mathrm{D}$ converter, and does not include errors in calculation of the least squares. Let $\epsilon_{1}$ refer to the sensitivity of the servo testing machine and $\epsilon_{2}$ the dissolving capability of $\mathrm{A} / \mathrm{D}$ converter in the maximum range of the load, both being non-dimensional. Then, the upper bound error, $\epsilon_{0}$, of the load is obtained from:

$$
\epsilon_{0}=\epsilon_{1}+\epsilon_{2} .
$$

Therefore, the parameter, $\epsilon$, adopted as a criterion of the unloading is given as shown in Fig. 16, and obtained from:

$$
\epsilon=\left\{\begin{array}{lr}
10 \epsilon_{0} & \text { for } P_{\boldsymbol{M}}>0.8 P_{d u} \\
\epsilon^{*}-\left(\epsilon^{*}-10 \epsilon_{0}\right) P_{\boldsymbol{M}} /\left(0.8 P_{a u}\right) \\
\text { for } 0.8 P_{a u} \geqq P_{\boldsymbol{M}} \geqq 0
\end{array}\right.
$$




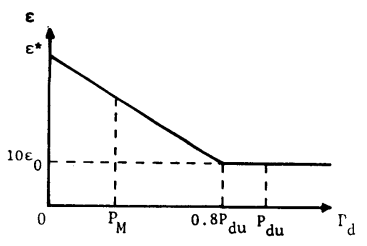

Fig. 16 Parameters of 1st Criterion for Unloading.

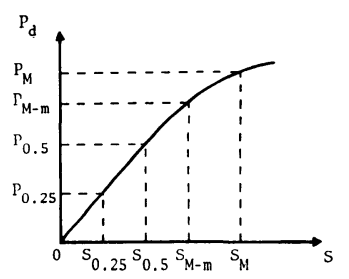

Fig. 17 Parameters of 2nd Criterion for Unloading.

where $P_{d u}$ represents the predicted maximum load on the destructive portion prescribed in the START program and $\epsilon^{*}$ is some constant value.

Since $\epsilon_{1}=0.001 \& \epsilon_{2}=0.005, \epsilon_{0}=0.006$. In the present tests, the value of $\epsilon^{*}$ was conveniently determined as $\epsilon^{*}=0.022$, and this was found to be quite satisfactory.

The criterion for the unloading is to exclude a casual erroneous reduction of the load generated by the action of the servo testing machine and A/D converter in lower range of the load.

(b) 2nd Criterion

A basic slope $\theta_{0}$, of the load, $P_{d}$, to the longitudinal displacement, $S$, indicated in Fig. 17, is defined as:

$$
\theta_{0} \equiv \frac{\left|P_{0.5}-P_{0.25}\right|}{\left|S_{0.5}-S_{0.25}\right|}
$$

where $P_{0.25}$ and $P_{05}$ refers to the value of the load, $P_{d}$, corresponding to $0.25 P_{d u}$ and $0.5 P_{d u}$, respectively. Similarily, $S_{0.25}$ and $S_{0.5}$ refers to the value of displacement, $S$, corresponding to the load $0.25 P_{d u}$ and $0.5 P_{d u}$, respectively.

Let $m$ and $\alpha$ be defined as an interval of the loading substep concerned and a parameter for the criterion, respectively. And let $\theta_{M}$ represent the slope defined by:

$$
\theta_{M} \equiv \frac{\left|P_{M}-P_{M-m}\right|}{\left|S_{M}-S_{M-m}\right|}
$$

where $M$ and $M-m$ refers to the current loading substep and the $m$-th previous substep, respectively.

Then, the second criterion for the unloading can be conveniently expressed by:

$$
\theta_{M} \leqq \frac{\theta_{0}}{\alpha} \quad \text { for } \quad P_{\boldsymbol{M}}>0.5 P_{d u}
$$

From the experience, $m$ and $\alpha$ was found most conveniently to be $m=5 \sim 7$ and $\alpha=8 \sim 32$, respectively. The choice of $m=5 \sim 7$ came from the considerations that both of the stroke displacement, $S_{\boldsymbol{M}}$, and the load, $P_{\boldsymbol{M}}$, undergo certain fluctuations, and the unduly local fluctuation of the slope, $\theta_{\boldsymbol{M}}$, should be avoided. Since the value of $\theta_{M}$ is so to speak, the average slope evaluated in terms of backward difference, and not being the one at the current point, $M$, assigning extremely small value of $\alpha$ does not necessarily guarantee the detection of the true instantaneous maximum load at point $M$. In most of the cases where rapid slope change was expected to occur, the value of $\alpha$ was taken to be 8 ; whereas, in some rare cases where only gradual change of the slope was expected, it was taken to be as small as 32 .

Whenever either of the above-mentioned criteria is satisfied, then, the function generator is caused to jump the program step forward for unloading by transmitting "FORWARD" signal through DIO interface. When the total load reaches the prescribed limit load, the loading step is considered to be completed, and the applied wave form is interrupted and held constant upon the detection of "HOLD" signal of DIO interface.

\section{Programmable Function Generator}

It has been explained that the function generator is used for loading and unloading the specimens so as to achieve the condition of either the constant stroke velocity or constant load rate by use of the ramp wave forms; moreover, it was also explained that the generator is subjected to the external control through DIO interface causing FORWARD, RESET, HOLD, and RUN.

\section{TEST SPECIMENS AND RESULTS}

The test specimens included steel stub columns of cruciforms, cylindrical shells, and boxes with or without a longitudinal rib, as illustrated in Fig. 13. The tests were conducted in the condition of either the constant load rate or the constant stroke velocity involving large repetitive axial deformations. Several examples of computer drawings will be provided herein.

Firstly, the load-displacement curves of a stub box column with a stiffener are obtained and plotted in Figs. 18 20. These figures consist of the following two types of relations: (a) load vs longitudinal displacement, and (b) load vs longitudinal average strain. Furthermore, Figs. 18 


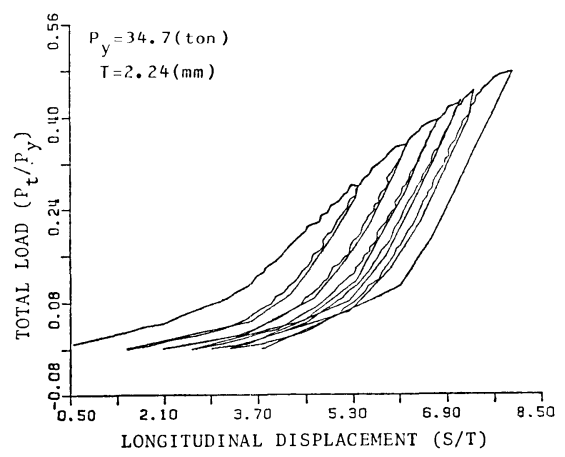

(a) Total Load vs. Longitudinal Displacement Curves.

Fig. 18

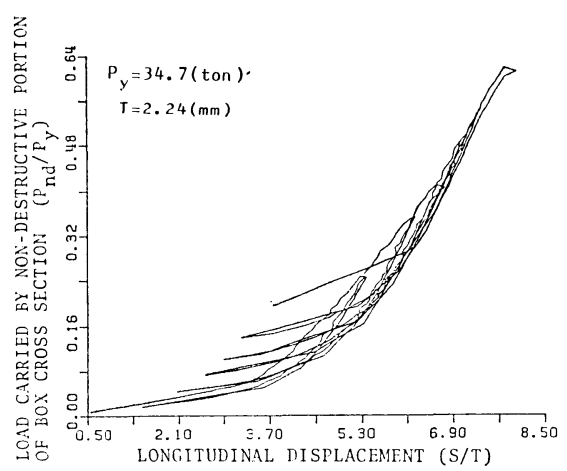

(a) Load Carried by Non-Destructives Portion vs. Longitudinal Displacement Curves.

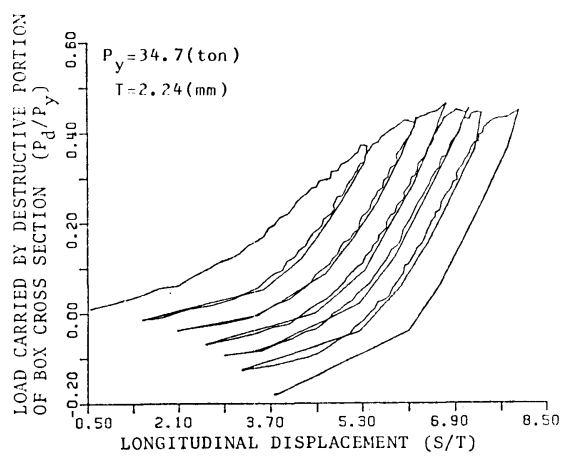

(a) Load Carried by Destructive Portion vs. Longitudinal Displacement Curves.

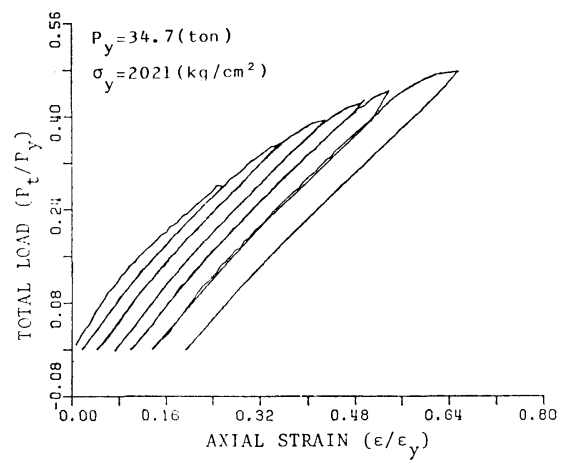

(b) Total Load vs. Longitudinal Curves.

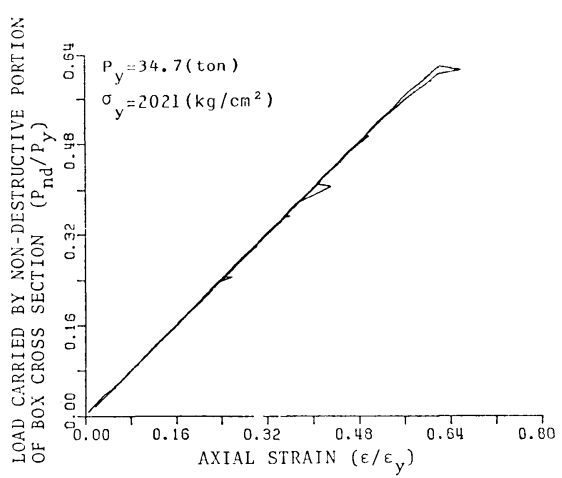

(b) Load Carried bv Non-Destructive Portion vs. Longitudinal Strain Curves.

Fig. 19

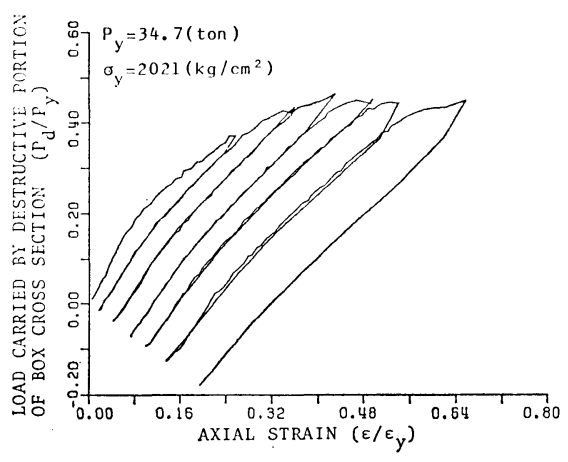

(b) Load Carried by Destructive Portion vs. Longitudinal Strain Curves..

Fig. 20

20 are concerned with the load corresponding to the total cross section, non-destructive portion, and the destructive portion, respectively. In the tests conducted so far, figures of the type (a), related with the destructive portion, like Fig. 20 (a) were chosen to be plotted "on-line". Comparing Fig. 18 with Fig. 20, it will be seen that the destructive portion has reached its local extremum load repeatedly; while, the total cross section has not reached its extremum load yet.

Further illustrations of plottings are provided in Figs. 21 23, which draw spatial distributions of the out-of-plane displacements of the speci- 


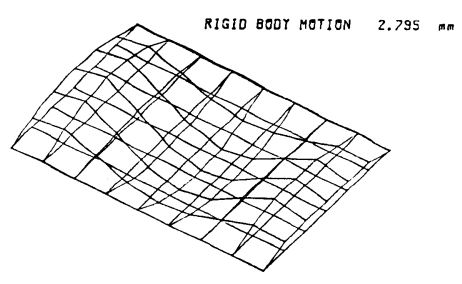

PLATE 1

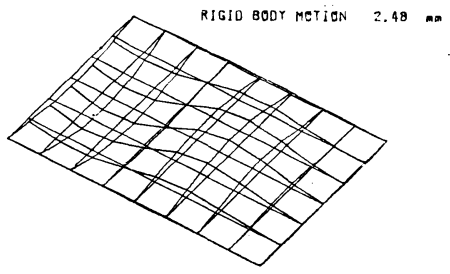

PLATE 3

BXOOA

STEP $=9$

(1: 13560$)$

Fig. 21 Deformations of Destructive Portion of a Stub Box Column.
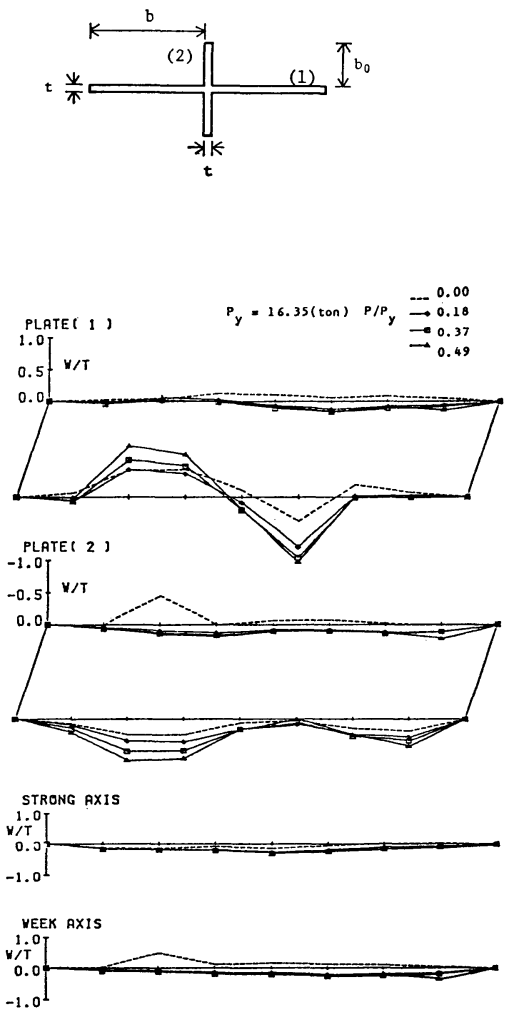

FiG. DEFLECTIONS OF PLATES \& COLUMN (CR32C)

Fig. 22 Deformations of Destructive Portion of a Stub Cruciform Column.
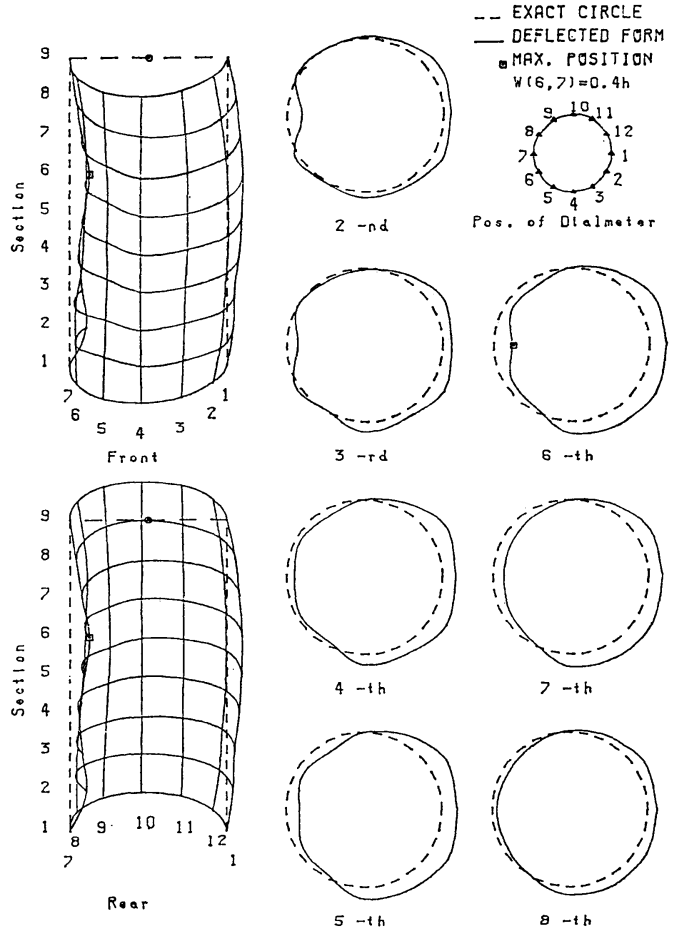

DEFLECTED FORM of SHZOC STEP $=0$

Flg. 23 Initial Deformations of a Cylindrial Shell.

mens acquired through GP-IB of the digital strain amplifier "off-line". Fig. 21 represents the deformations of the destructive portion of a stub box column without a stiffener under loading. On the other hand, Fig. 22 represents the deformations of the destructive portion (1), and the non-destructive portion (2) of a cruciform. It maybe seen that the destructive portion (a) deformed torsionally.

The initial displacements were plotted "online" together with the corresponding residual displacements. Fig. 23 shows an example of such plottings and is concerned with a cylindrical shell indicating both longitudinal and circumferential configurations.

After a series of tests, the following observations have been made:

(a) The local extremum loads may be deemed to have been detected by many dynamic strain amplifiers, AIO interface, and the real-time decision making by a microcomputer.

(b) A special rig was designed to measure digitally the deflectional wave form of the specimen, which was proved to be quite efficient.

(c) The use of a programmable function generator which is a slave computer, externally 
controllable was proved feasible as an excellent wave from synthesizer for the servo testing machine.

(d) The unloading criteria based on the load vs axial displacement relationship may be found to be satisfactory. For example, the choice of $m=$ $5 \sim 7, \alpha=8 \sim 32$, and $\epsilon=10 \epsilon_{0}$, in Eq. (7), Inequality (8), and Inequality (5), respectively, was found quite satisfactory.

(e) The current microcomputer system with the main memory of 64-kbyte RAM and external memory of $0.7 \mathrm{M}$ byte is found just satisfactory. However, if more complex specimen is to be tested, the more external memory will be required.

\section{CONCLUSIONS AND ACKNOWLEDGE- MENT}

In this report, a new automated testing system with the combination of two microcomputers, one being the master controller, the other being the slave function generator, is proposed. The system is composed of three groups: Servo testing machine group, Data acquisition group, and Microcomputer group. The system has been applied to a series of buckling tests on thin-walled steel structural members under repetitive loading, and has the following advantages: quick real-time acquisition and computation of large quantities of data for swift decision making on the continuously varying state of the test specimen, quick digital feedback control of servo testing machine, efficient measurement of deflectional wave form of the specimen, real-time display of the data, efficient storage of data, and smooth transmission of data to a larger data processing center via intelligent TSS terminal.

The authors admit that there may be some other types of loading other than the ramp wave form to find the basic strength characteristics of thin-walled structures, however, no matter what they are, the authors believe in the key role played by microcomputers. Furthermore, they believe also that the test automation can be better accomplished with a multi-microcomputer system rather than with a single minicomputer, and that the use of GP-IB will become increasingly popular.

The general concepts adopted herein may be easily extended and applicable to other types of automated structural testing, and without doubt, the computerized system like what has been presented herein will be adopted as the standard testing procedure. Although a single program- mable function generator was used as a slave processor herein, several versatile microcomputers interconnected by GP-IB may be used as slave computers in future.

The authors express appreciation toward Professor N. Shiraishi of Kyoto University for the encouragement of the study and every convenience he offered to them. This study accepted a Grant-in-Aid for Developmental Scientific Research from the Ministry of Education, Science and Culture in years 1981-1982.

\section{BIBLIOGRAPHY}

1) Tomonaga, K. and Y. Maeda: Some basic consideration in erection procedures of steel bridges, Proc. JSSC, Vol. 10, No. 105, 1974, pp. 9-33 (in Japanese).

2) Niwa, Y., E. Watanabe and Y. Iwashimizu: On the initial imperfections and their relations to the strength of web-plates of actual steel bridges, Memoirs of Faculty of Engineering, Kyoto Univ., Vol. 43, Part 2, pp. 240-264, 1981.

3) Komatsu, S., Y. Niwa and E. Watanabe: Statistical study on imperfections of steel webs, Journal of Structural Division, ASCE, (to appear in April 1983).

4) Bolotin, V. V.: Statistical methods in structural mechanics, Holden-Day, Inc., San Francisco, 1969.

5) Yamada, Y. and H. Iemura: Hybrid analysis on earthquake response of deteriorating hysteretic structures, Proc. of the Sino-American Symp. on Bridge \& Str. Eng., Peking, 1982.

6) Ramirez, E. V. and M. Weiss: Microprocessing Fundamentals-Hardware and Software, McGraw-Hill, Inc., 1980.

7) SORD Computer System: Microcomputer M200 mark II series, 1979 (in Japanese).

8) Hiramatsu, K. and T. Saito: Interfaces of microcomputer, Ohmu-sha, 1979 (in Japanese).

9) SORD Computer System: M200 mark series I/O interface, 1981 (in Japanese).

10) Kashida, M.: GPIB controller system of readout devices by personal computer PC-8001, Denshitembo, Vol. 19, No. 1, 1981 (in Japanese).

11) Okumura, M.: IEEE-488 standard digital bus and its applications, Interface, Feb., 1979 (in Japanese).

12) Katayama, A.: On GP-IB (General Purpose Interface Bus), TEAC Technical Report 1, 1981 (in Japanese).

(Received March 26, 1982) 